\title{
COMMENT
}

\section{A new paradigm in bench to bedside research, with a stop in the dusty pharmaceutical cabinet?}

\author{
Vivek Balasubramaniam ${ }^{1}$, Lois K. Lee ${ }^{2}$ and Joyce R. Javier ${ }^{3}$ \\ Pediatric Research (2021) 89:1604-1605; https://doi.org/10.1038/s41390-021-01482-x
}

In the past year, we have all suffered and survived through an event-the COVID-19 pandemic - that will shape our future as a society and have far-ranging influences that include political, economic, and scientific practices. As future scientists look back at this event, will they point to this as the inflection point where it became apparent that through a coordinated and collaborative approach the human race was able to work together in order to restrain the outbreak of a deadly pandemic? Or will they look regretfully at what could have been if we lapse back into the secretive, individualistic approach that has been the norm of science up to this point?

In the past year, we have stood witness, in some cases have been active participants, in a collaborative endeavor. This includes the identification of a new pathogen, decryption of its genetic code, interpretation of its mode of action in order to devise treatments (often repurposing existing therapies), and the successful development of (multiple) vaccines utilizing a novel approach that went from theory to validated treatment in $<1$ year. The teaming up of multiple disciplines, from basic scientists, geneticists, biostatisticians, chemists, translational researchers, clinical investigators, government regulatory agencies, pharmaceutical companies, and clinical trials organizations, with a singular purpose of understanding a novel disease and developing a preventive vaccine has never been witnessed before in history. While this unified pursuit of a viable vaccine to protect against COVID-19 proceeded at an astonishing pace, we witnessed an overwhelmed medical community share data and perspective in "real time" on the success and failures of approaches to treating the clinical syndrome that cost so many lives to this viral pandemic. In early 2020, new approaches and therapies were flying around like a tornado, with desperate clinicians clinging to any suggestion of an effective treatment to check the deadly denouement of COVID-19 infection. As they shared their experiences, from places like China, Italy, Vancouver, Seattle, and New York, new strategies emerged. These included the repurposing of immune-modulating antibodies (e.g., anti-IL-6), to the rescue of heretofore unsuccessful therapies (e.g., remdesivir), and the realization of established medications (e.g., dexamethasone), as having a significant benefit. Many of these therapies had been sitting on our "shelf"-pharmaceuticals gathering dust. These treatments would not have been utilized if not for the free sharing of information, open (and sometimes vigorous) debate, willingness to quickly overcome what were thought of as "insurmountable" barriers to conducting efficient clinical studies, the embrace of the academic medical community to "pre-publication" of findings, and the acknowledgment of the considerable worth of publication of "negative" studies.

The understanding of the pathogenesis and path to mortality occurring with COVID-19 infection was hastened by the collection of clinical samples from those who succumbed to the disease, in addition to those who survived. The analysis of the blood, tissue, and secretions of these individuals directed skillful scientists and clinicians towards approaches to treat this devastating viral infection. In this issue of Pediatric Research, Dapat et al. ${ }^{1}$ in their article "Gene signature of children with severe respiratory syncytial virus infection" provide us with a roadmap, which can be followed by clinicians and scientists to look for novel treatments for another virus with particular morbidity and mortality in the pediatric age group. In their manuscript, they have analyzed the genetic expression pattern in children with severe respiratory syncytial virus (RSV) and used this to identify 43 potentially effective drugs for the treatment of RSV. Of these drugs, one-third are already approved by the FDA and one-quarter are currently under investigation for other diseases.

If we have truly learned lessons of cooperation and collaboration from the researcher to the government regulator to the clinician, could we develop new approaches with existing therapies to treat RSV and other diseases that afflict and kill our children? This would require the open sharing of data including facilitating forums open to clinicians, scientists, and the pharmaceutical industry to discuss results, propose therapies, and study design. For example, in order to make data, protocols, and procedures available for collaboration, the NIH has a current policy for data sharing (https://grants.nih.gov/policy/sharing. $\mathrm{htm})$. Although multidisciplinary programs in pediatric research exist, the time it takes to develop a therapy from bench to bedside to community is $>10$ years. In comparison, the advancements made in $<1$ year in response to COVID-19 suggest that an interdisciplinary or transdisciplinary approach should be followed. Rosenfield ${ }^{2}$ described a three-tiered structure to conceptualize the type of collaboration among different disciplines: (i) level one is a multidisciplinary approach, by far the most common approach where researchers work in parallel or sequentially from a disciplinary-specific base to address a common problem; (ii) level two is an interdisciplinary approach where work is done jointly, but still from a disciplinaryspecific basis to address a common problem; (iii) finally, a

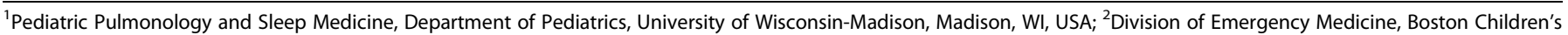

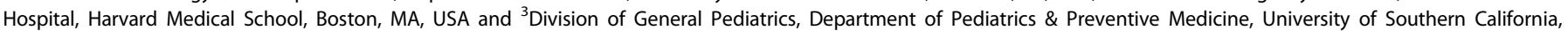
Children's Hospital Los Angeles, Keck School of Medicine, Los Angeles, CA, USA

Correspondence: Vivek Balasubramaniam (Balasubramaniam@pediatrics.wisc.edu)

Received: 2 March 2021 Accepted: 7 March 2021

Published online: 6 April 2021 
transdisciplinary approach sees researchers working jointly using a shared conceptual framework breaking down partitions and drawing together disciplinary-specific theories, concepts, and approaches to address a common problem. One may be quick to point out that the enormous amount of funding that was devoted to this singular purpose was behind the success, but that would not acknowledge the power of the removal of partitions between disciplines that resulted in large-scale transdisciplinary research that is responsible for the development of successful therapies in response to a global pandemic. This would require funding agencies to acknowledge the benefit of encouraging such collaborations. Beyond the academic community, clinical data, study products, and other resources should be shared with community agencies, patient and parent advocacy groups, policymakers, and the public in order to translate research into practice and policy. Finally, it will require academic institutions to acknowledge the value of interdisciplinary and transdisciplinary teams and reevaluate their emphasis on first and senior authorship and give equal academic credit (e.g., for promotion and tenure) to all participants in such endeavors. Are we ready for this paradigm shift? Are we living during an inflection point representing a revolutionary change in the treatment of pediatric diseases? What say you, Pediatric Researchers, are you able to change your approach? Are you up to this challenge to advance medicine for the good of today's and tomorrow's children?

\section{ADDITIONAL INFORMATION}

Competing interests: The authors declare no competing interests.

Publisher's note Springer Nature remains neutral with regard to jurisdictional claims in published maps and institutional affiliations.

\section{REFERENCES}

1. Dapat, C. et al. Gene signature of children with severe respiratory syncytial virus infection. Pediatr. Res. (2021).

2. Rosenfield, P. L. The potential of transdisciplinary research for sustaining and extending linkages between the health and social sciences. Soc. Sci. Med. 35, 1343-1357 (1992). 